\title{
A comparative study to assess the safety and efficacy of etoricoxib versus aceclofenac in osteoarthritis
}

\author{
Harsimrat S. Waraich, Vikas Kumar*, Ashok Goel, Jatinder Singh
}

Department of Pharmacology, Government Medical College, Amritsar, Punjab, India

Received: 07 August 2018

Accepted: 31 August 2018

*Correspondence to:

Dr. Vikas Kumar,

Email: kumar.vikas444@

gmail.com

Copyright: (C) the author(s), publisher and licensee Medip Academy. This is an openaccess article distributed under the terms of the Creative Commons Attribution NonCommercial License, which permits unrestricted noncommercial use, distribution, and reproduction in any medium, provided the original work is properly cited.

\begin{abstract}
Background: Osteoarthritis (OA) is most common form of arthritis; also referred as degenerative joint disease or "wear and tear" arthritis. Cyclooxygenase-2 (COX-2) inhibitors are effective for pain and inflammation in OA and gained importance over conventional non-steroidal anti-inflammatory agents (NSAIDs), as causes significantly less toxicity, particularly, in gastrointestinal tract. The objective of the present research was to study the short-term comparative clinical efficacy of aceclofenac and etoricoxib in patients with osteoarthritis and to compare the safety profile of the two drugs i.e. aceclofenac and etoricoxib.

Methods: The present study was a prospective, open label, parallel, intention to treat 80 patients out of 102 screened for osteoarthritis in the Department of Orthopaedics, Guru Nanak Dev Hospital attached to the Government Medical College, Amritsar. Patients were randomly divided in two groups with 40 patients each. Group A patients received Tab etoricoxib 60mg once daily and Group B patients received Tab. Aceclofenac 100mg twice daily. Patients were followed up after three weeks and at six weeks for clinical efficacy and safety.

Results: Both the groups found to have significant improvement in signs and symptoms of osteoarthritis. However, aceclofenac was superior to etoricoxib in terms of change in visual analogue scale score, osteoarthritic severity index, patients' and physicians' global assessment while, etoricoxib was superior in terms of WOMAC osteoarthritic index and safety parameters in terms of ADR. Conclusions: Etoricoxib was better than conventional NSAIDs for the symptomatic management of osteoarthritis in terms of safety profile and clinical efficacy.
\end{abstract}

Keywords: Aceclofenac, Etoricoxib, Osteoarthritis, VAS, WOMAC

\section{INTRODUCTION}

Osteoarthritis (OA) is most common form of arthritis; also referred as degenerative joint disease or "wear and tear" arthritis. ${ }^{1}$ It is usually associated with aging and most likely affect the joints that are continually stressed throughout the years including knees, hips, fingers, and lower spine region. It is one of the ten most disabling disease in developed countries. ${ }^{2}$ Almost $9.6 \%$ of men and $18.0 \%$ of women aged over 60 years, globally, have symptomatic osteoarthritis. ${ }^{2}$ It affects about $4-6 \%$ of adult population and is mentioned one of the top five chronic disease in India. ${ }^{3}$ The goal of osteoarthritis treatment is to reduce pain and improve function as there is no cure for disease, but some treatment attempts to slow the disease progression. ${ }^{4}$ Most often, the combination of therapies include physical measures, drug therapy and, sometimes, surgery. ${ }^{4}$

Pharmacotherapy for $\mathrm{OA}$ is focused mainly to alleviate pain, maintenance of quality of life and preservation of functional independence. At present, there is no pharmacological agents capable of retarding the progression or preventing OA. ${ }^{5}$ Pharmacological agents for treatment of OA are, oral acetaminophen, oral and topical non-steroidal anti-inflammatory drugs (NSAIDs), oral selective cyclooxygenase-2 (COX-2) inhibitors, 
opiates, topical capsaicin, intraarticular injections of steroids and hyalronans. ${ }^{6}$

NSAIDs are mainstay for initial symptomatic treatment of OA. The mechanism by which NSAIDs exerts antiinflammatory and analgesics effects is by inhibition of prostaglandin (PG) generating enzymes, cyclooxygenase (COX). In addition to inflammatory potential, PGs also contribute to important hemostatic functions such as, maintenance of gastric lining, renal blood flow and platelet aggregation. The conventional non-selective NSAIDs (ibuprofen, Naprosyn, indomethacin) by reducing the PG level in these organs can lead to side effects like gastric ulceration, renal insufficiency and prolonged bleeding time. ${ }^{7}$ Therefore, efficacy and toxicity of NSAIDs results from their inhibition of COX, which primarily has two structurally and functionally distinct isoforms, $\mathrm{COX}-1$ and COX-2. COX-1 is the constitutive isoform expressed throughout the body and has important role in gastrointestinal protection and platelet aggregation. Whereas COX-2 is inducible that is involved in inflammatory response. ${ }^{8}$ The discovery of COX-2 led to development of therapeutic COX-2 inhibitors based on the hypothesis that they would afford efficacy similar to traditional NSAIDs and with better gastro-intestinal (GI) tolerability. $^{7}$

Cyclooxygenase-2 (COX-2) inhibitors are effective for pain and inflammation in OA and gained importance over conventional non-steroidal anti-inflammatory agents (NSAIDs), as causes significantly less toxicity, particularly, in gastrointestinal tract. ${ }^{7}$ The potential clinical benefit of selective COX-2 inhibitors are significant in management of chronic pain in OA. ${ }^{9}$ COX-2 inhibitors initially approved were; celecoxib, rofecoxib, valdecoxib and its pro-drug parecoxib, etoricoxib and lumiracoxib. Most coxibs are either severely restricted in their use or withdrawn from the market in view of their adverse event profile. $^{7}$ A systematic review of randomized controlled trials on safety, efficacy and tolerability of COX-2 for treatment of OA has shown to be significantly improved GI safety and tolerability. ${ }^{10}$

The safety and efficacy of etoricoxib 30mg and celecoxib $200 \mathrm{mg}$ in the treatment of osteoarthritis in two identically designed, randomized, placebo-controlled, non-inferiority studies had similar efficacy and both had similar safety in treatment of knee and hip OA versus placebo. ${ }^{11}$ The long term efficacy and tolerability of etoricoxib over 52 weeks during treatment of OA patients, in a double-blind, randomized, multicenter, active-comparator controlled trial; demonstrated maintenance significant clinical efficacy at dose ranging from 90-120mg. ${ }^{12}$ The efficacy and safety of etoricoxib compared with naproxen in two, 138 weeks randomized studies of patient with osteoarthritis, was found to be clinically efficacious and well tolerable. ${ }^{13}$ A randomized placebo-controlled trial, comparing the efficacy of etoricoxib $30 \mathrm{mg}$ and ibuprofen $2400 \mathrm{mg}$ ( $800 \mathrm{mg}$ thrice daily), for 12 weeks in the patients of $\mathrm{OA}$, demonstrated superior efficacy of etoricoxib versus placebo and comparable clinical efficacy versus ibuprofen. ${ }^{14}$ In a randomized placebo-controlled trial, comparing the efficacy of etoricoxib, celecoxib, lumiracoxib, non-selective NSAIDs and acetaminophen for the treatment of $\mathrm{OA}$, found that etoricoxib $30 \mathrm{mg}$ resulted in greatest improvement in pain and physical function. ${ }^{15}$

The present study was conducted to assess the safety and efficacy of etoricoxib versus aceclofenac (conventional Non-steroidal anti-inflammatory drug) especially in the north Indian population mainly in and around the city of Amritsar.

The objective of the present research was to study the short-term comparative clinical efficacy of aceclofenac and etoricoxib in patients with osteoarthritis and to compare the safety profile of the two drugs i.e. aceclofenac and etoricoxib.

\section{METHODS}

The present study was a prospective, open label, parallel, intention to treat 80 patients of osteoarthritis visiting the OPD/Wards of the Department of Orthopaedics, Guru Nanak Dev Hospital attached to the Government Medical College, Amritsar. This study was conducted in accordance with the principles of good clinical practice and the Decleration of Helsinki.

\section{Inclusion criteria}

- Patients with symptomatic osteoarthritis of age 35 to 60 years who fulfil American College of Rheumatology criterion for the diagnosis of osteoarthritis were enrolled

- Patients having symptomatic osteoarthritis either as a new case or as an old case following discontinuation of treatment with NSAIDS or other analgesic medications with a washout period of one week

- All these patients were examined as per the baseline criterion and were included in the study if they fit this criterion.

\section{Exclusion criteria}

- Age less than 35 years or more than 60 years

- The patients who present with active concomitant gastroduodenal disorders, hepatic and renal impairment within last 30 days prior to receiving the study drug

- Patient with active cardiac lesion or a known hypertensive patient

- Pregnant females or those who were planning their pregnancy during the study

- Patients who had received oral, intramuscular, intraarticular or soft tissue injections of corticosteroids within last four weeks before receiving the first dose of the study medication 
- Patients diagnosed to have any inflammatory arthritis, gout or acute trauma of the knee, hip or spine

- Patients with a known hypersensitivity to NSAIDS

- Patients with any known contraindication of the said drugs

- Female patients on oral contraceptive pills.

The approval from Institutional ethics committee, Government Medical College, Amritsar was obtained. Informed consent was sought from all the patients included in the present study.

Patients were randomly divided into 2 groups, A and B, consisting of 40 patients each. The duration of the study was six weeks. Group A patients received Tab Etoricoxib 30mg once daily and Group B patients received Tab. Aceclofenac 100mg. B.D. Patients were followed up after three weeks and at six weeks for clinical efficacy and safety.

Screening or baseline clinical assessment of osteoarthritis included patient's assessment of arthritic pain on a Visual Analogue Scale (VAS), the osteoarthritis severity index, on a scale of 0-24 based upon the Western Ontario and Macmaster Universities (WOMAC) osteoarthritis index, patients and physician's global assessment of osteoarthritis.

\section{Baseline investigations}

The investigation that were carried out were:

- $\quad$ X-ray of both the knees AP view and lateral view was taken to corroborate the diagnosis of osteoarthritis at day 0 and six weeks.
- Stool for occult blood was done at day 0, 21 and 42 .

- $\quad$ ECG was done at day 0, 21 and 42.

\section{Statistical analysis}

The observations were tabulated in the form of mean \pm standard deviation and analysis was done using student's " $t$ " test and level of significance was determined as its ' $p$ ' value with $\mathrm{p}<0.05$ taken as statistically significant.

\section{RESULTS}

A total of 102 patients were screened for the study from the O.P.D. of Department of Orthopaedics, Government Medical College, Amritsar.

Twelve patients did not meet the inclusion criterion. 10 patients did not complete the study. Out of the remaining 80 patients, they were divided into two groups of 40 each and they were followed up for 6 weeks (Table 1).

Table 1: Base line characteristics of study population.

\begin{tabular}{|lll|}
\hline Demographic parameters & Group A & Group B \\
\hline Age & $55.97 \pm 1.77$ & $55.42 \pm 1.21$ \\
\hline Female:Male & $28: 5$ & $26: 7$ \\
\hline Height & $5.42 \pm 3.73$ & $5.41 \pm 4.31$ \\
\hline Weight & $66.33 \pm 1.81$ & $66.48 \pm 1.29$ \\
\hline Medical history & \\
\hline Duration of illness (months) & $4.91 \pm 0.72$ & $4.96 \pm 0.40$ \\
\hline
\end{tabular}

Table 2: Visual Analogue Scale.

\begin{tabular}{|llllll|}
\hline & Basal & 3 weeks & Change & 6 weeks & Change \\
\hline Group A & $78.18 \pm 1.28$ & $41.60 \pm 1.6$ & $37.12 \pm 1.7$ & $25.15 \pm 1.47$ & $53.03 \pm 1.90$ \\
\hline Group B & $81.47 \pm 1.05$ & $27.06 \pm 1.16$ & $54.41 \pm 1.38$ & $10.44 \pm 0.85$ & $71.02 \pm 0.91$ \\
\hline
\end{tabular}

Table 3: WOMAC osteoarthritis index score.

\begin{tabular}{|llllll|}
\hline & Basal & 3 weeks & Change & 6 weeks & Change \\
\hline Group A & $65.38 \pm 1.38$ & $27.68 \pm 0.92$ & $24.93 \pm 1.93$ & $9.00 \pm 0.85$ & $56.38 \pm 1.22$ \\
\hline Group B & $59.94 \pm 2.41$ & $35.00 \pm 1.62$ & $24.93 \pm 1.93$ & $19.85 \pm 1.54$ & $40.09 \pm 2.06$ \\
\hline
\end{tabular}

Table 4: Osteoarthritic severity index.

\begin{tabular}{|llllll|}
\hline & Basal & 3 weeks & Change & 6 weeks & Change \\
\hline Group A & $22.09 \pm 0.81$ & $21.70 \pm 0.97$ & $0.39 \pm 0.22$ & $13.88 \pm 0.83$ & $8.21 \pm 0.76$ \\
\hline Group B & $23.76 \pm 0.17$ & $23.29 \pm 0.49$ & $0.47 \pm 0.38$ & $7.91 \pm 0.86$ & $15.85 \pm 0.85$ \\
\hline
\end{tabular}

Table 5: Patient global assessment.

\begin{tabular}{|llllll|}
\hline & Basal & 3 weeks & Change & 6 weeks & Change \\
\hline Group A & $1.30 \pm 0.08$ & $3.55 \pm 0.12$ & $2.24 \pm 0.13$ & $3.97 \pm 0.13$ & $2.66 \pm 0.15$ \\
\hline Group B & $1.24 \pm 0.07$ & $4.30 \pm 0.07$ & $3.16 \pm 0.12$ & $4.90 \pm 0.05$ & $3.66 \pm 0.13$ \\
\hline
\end{tabular}




\section{Efficacy assessments}

\section{Visual Analogue Scale}

Etoricoxib in dose of 30mg once daily and aceclofenac in the dose of $100 \mathrm{mg}$ twice daily produced significant improvement in signs and symptoms of osteoarthritis as determined by all the measures of efficacy.

Etoricoxib in dose of $30 \mathrm{mg}$ once daily and aceclofenac in the dose of $100 \mathrm{mg}$ twice daily produced significant decrease on scores on visual analogue scale. Etoricoxib reduced the score to $41.60 \pm 1.6$ in 3 weeks and to $25.15 \pm 1.47$ in 6 weeks as compared baseline score of $78.18 \pm 1.28(\mathrm{p}<0.001)$. Aceclofenac reduced the score to $27.06 \pm 1.16$ in 3 weeks and to $10.44 \pm 0.85$ in 6weeks as compared to basal score of $81.47 \pm 1.05$ ( $p<0.001$ ). Acelofenac produced a significantly better change in score. Both the treatments produced significant relief of pain, aceclofenac being superior to etoricoxib (Table 2).

\section{WOMAC osteoarthritic index}

Etoricoxib in the dose of 30mg once daily and Aceclofenac $100 \mathrm{mg}$ twice daily produced a significant decrease in the score of WOMAC osteoarthritic index indicating a significant reduction in pain and stiffness and an improvement in physical functioning. Aceclofenac in the dose of $100 \mathrm{mg}$ twice daily significantly reduced the basal score of $59.94 \pm 2.41$ to $35 \pm 1.62$ in 3 weeks and further reduction in the score to $19.85 \pm 1.54$ in 6 weeks $(\mathrm{p}<0.001)$. Etoricoxib in the dose of $30 \mathrm{mg}$ once daily reduced the score to $27.68 \pm 0.92$ in 3 weeks and to $9.00 \pm 0.85$ in 6weeks from a basal level of $65.38 \pm 1.38(\mathrm{p}<0.001)$. Thus, both the groups produced a significant relief of pain, but with etoricoxib the response was significantly better $(\mathrm{p}<0.01)$ (Table 3).

\section{Osteoarthritis severity index}

Etoricoxib in the dose of $30 \mathrm{mg}$ once daily and aceclofenac $100 \mathrm{mg}$ twice daily produced a significant decrease in osteoarthritic severity index. Etoricoxib reduced the basal score from $22.09 \pm 0.81$ to $21.70 \pm 0.97$ at 3 weeks and $13.88 \pm 0.83$ at 6 weeks $(\mathrm{p}<0.001)$ aceclofenac reduced the basal score from $23.76 \pm 0.17$ to $23.29 \pm 0.49$ at 3 weeks and $7.91 \pm 0.86$ at 6 weeks $(\mathrm{p}<0.001)$. Thus, both etoricoxib in the dose of $30 \mathrm{mg}$ once daily and aceclofenac $100 \mathrm{mg}$ twice daily produced a significant decrease in signs and symptoms but aceclofenac was found to be slightly better (Table 4).

\section{Patient's global index}

Etoricoxib in the dose of 30mg once daily and aceclofenac in the dose of $100 \mathrm{mg}$ twice daily produced a significant increase in the scores of patients' global assessment implying a significant improvement in signs and symptoms. Etoricoxib increased the score from a basal score of $1.30 \pm 0.08$ to $3.55 \pm 0.12$ at 3 weeks and $3.97 \pm 0.13$ at 6 weeks $(\mathrm{p}<0.01)$. Aceclofenac increased the basal score of $1.24 \pm 0.07$ to $4.30 \pm 0.07$ at 3 weeks and $4.90 \pm 0.05$ at 6 weeks $(\mathrm{p}<0.01)$ thus improvement in patient global index was significantly better with aceclofenac than with etoricoxib (Table 5).

\section{Physicians global assessment}

Etoricoxib in the dose of $30 \mathrm{mg}$ once daily and aceclofenac in the dose of $100 \mathrm{mg}$ twice daily produced a significant increase in the scores of physicians' global assessment implying a significant improvement in signs and symptoms. Etoricoxib increased the score from a basal score of $1.21 \pm 0.07$ to $3.58 \pm 0.14$ at 3 weeks and $4.00 \pm 0.15$ at 6 weeks $(\mathrm{p}<0.01)$. Aceclofenac increased the basal score of $1.65 \pm 0.17$ to $4.52 \pm 0.08$ at 3 weeks and $4.91 \pm 0.04$. So, there was significantly better increase in physicians' global index in patients taking aceclofenac as compared to etoricoxib (Table 6).

\section{Safety assessment}

\section{Gastrointestinal tract related adverse effects}

Gastrointestinal tract (GIT) related adverse effects were mostly found to be mild to moderate in nature. These were reported in $7.5 \%$ of patients taking aceclofenac. Patients had problems of abdominal pain, diarrhoea, dyspepsia, flatulence and nausea. Whereas no adverse events were reported by patients taking etoricoxib (Table 7).

Table 6: Physicians global index.

\begin{tabular}{|llllll|}
\hline & Basal & 3 weeks & Change & 6 weeks & Change \\
\hline Group A & $1.21 \pm 0.07$ & $3.58 \pm 0.14$ & $2.36 \pm 0.10$ & $4.00 \pm 0.15$ & $2.78 \pm 0.13$ \\
\hline Group B & $1.65 \pm 0.17$ & $4.52 \pm 0.08$ & $3.02 \pm 0.20$ & $4.91 \pm 0.04$ & $3.26 \pm 0.17$ \\
\hline
\end{tabular}

\section{Adverse events other than GIT}

Only one patient was reported to have rash in the group treated with aceclofenac. Etoricoxib was found to be better tolerated as compared to aceclofenac (Table 7).

\section{DISCUSSION}

Non-steroidal anti-inflammatory drugs are widely used for the relief of pain, stiffness and joint swelling in osteoarthritis and rheumatoid arthritis. 
Table 7: Incidence of adverse effects during the study period.

\begin{tabular}{|c|c|c|}
\hline Adverse effects & $\begin{array}{l}\text { Group A } \\
\text { (Etoricoxib) }\end{array}$ & $\begin{array}{l}\text { Group B } \\
\text { (Aceclofenac) }\end{array}$ \\
\hline \multicolumn{3}{|c|}{ GIT adverse effects } \\
\hline Abdominal pain & - & $3(7.5 \%)$ \\
\hline Diarrhoea & - & $3(7.5 \%)$ \\
\hline Dyspepsia & - & $3(7.5 \%)$ \\
\hline Flatulence & - & $3(7.5 \%)$ \\
\hline Nausea & - & $3(7.5 \%)$ \\
\hline \multicolumn{3}{|c|}{ Non-GIT adverse effects } \\
\hline Skin rash & - & $1(2.5 \%)$ \\
\hline
\end{tabular}

Although NSAIDs are generally well tolerated, upper gastrointestinal adverse effects dyspepsia, nausea and abdominal pain, mucosal lesions and serious complications such as bleeding and perforation are associated with their use. As the adverse effects of conventional NSAIDs are mainly due to inhibition of COX-1 while therapeutic effects result from COX-2 inhibition. So, drugs which are selective COX-2 inhibitors seem to be promising alternatives in patients intolerant to conventional NSAIDs.

Etoricoxib, selective COX-2 inhibitor, has shown promising role for symptomatic relief from pain and inflammation in patients of OA without any gastrointestinal adverse effect. Many studies have demonstrated that etoricoxib is equally efficacious and has better safety profile as compared to conventional NSAIDs. ${ }^{15}$

So, the present study was conducted to assess the safety and efficacy etoricoxib versus aceclofenac, a commonly used conventional non-steroidal anti-inflammatory drug in the patients coming to the out-patient department, Department of Orthopaedics, Guru Nanak Dev Hospital, Government Medical College, Amritsar.

Etoricoxib is recommended in a dose of 30mg once daily in patients of osteoarthritis. For aceclofenac, the standard dose which is recommended is $100 \mathrm{mg}$ twice daily. Both etoricoxib and aceclofenac produced significant improvement in signs and symptoms of osteoarthritis as determined by all the measures of efficacy.

Both etoricoxib and aceclofenac produced significant decrease in scores on visual analogue scale. In a randomized controlled trial, comparing the efficacy of aceclofenac and etoricoxib in pain control over baseline on visual analogue scale, documented to have statistically significant results in both the treatment groups. ${ }^{16}$ However, in present study, both the treatments produced significant relief of pain, aceclofenac being superior to etoricoxib in terms of change in visual analogue scale score.

Etoricoxib in the dose of 30mg once daily and aceclofenac $100 \mathrm{mg}$ twice daily produced a significant decrease in the score of WOMAC osteoarthritic index indicating a significant reduction in pain and stiffness and an improvement in physical functioning; with response to etoricoxib better as compared to aceclofenac. In a randomized, double blind, placebo and active comparatorcontrolled trial conducted in 617 adults with knee OA, etoricoxib was more effective than placebo after six weeks as measured by WOMAC pain sub-scale. ${ }^{17}$

Etoricoxib in the dose of $30 \mathrm{mg}$ once daily and aceclofenac $100 \mathrm{mg}$ twice daily produced a significant decrease in osteoarthritic severity index; but aceclofenac was found to be slightly better. In a systematic review including nine prospective studies, comparing the safety, efficacy and tolerability, aceclofenac was superior in decreasing osteoarthritis severity index. ${ }^{18}$

Etoricoxib in the dose of 30mg once daily and aceclofenac in the dose of $100 \mathrm{mg}$ twice daily produced a significant increase in the scores of patients' global assessment implying a significant improvement in signs and symptoms; with aceclofenac better than etoricoxib. In a double blind randomized controlled trial conducted for six weeks to evaluate efficacy and tolerability of aceclofenac for the treatment of symptomatic OA patients, showed significant improvement in patients' global assessment of disease activity. ${ }^{19}$

Etoricoxib in the dose of 30mg once daily and Aceclofenac in the dose of $100 \mathrm{mg}$ twice daily produced a significant increase in the scores of physicians' global assessment implying a significant improvement in signs and symptoms, with aceclofenac showing significantly better improvement in physicians' global index. In a double blind randomized controlled trial conducted for six weeks to evaluate efficacy and tolerability of aceclofenac for the treatment of symptomatic OA patients, showed significant improvement in physicians' global assessment of disease activity. ${ }^{19}$

GI related adverse drug reactions (ADR) were reported in the group taking aceclofenac while no such ADR reported in patients on etoricoxib. Thus, etoricoxib showed better GI tolerability as compared to aceclofenac. In a randomized, double blind trial, conducted to compare the GI tolerability, safety and efficacy of etoricoxib in patients' with OA, it was associated with significantly better GI tolerability. ${ }^{20}$

Limitations of the present research was a study involving 80 patients and no placebo control was used in this study in view of the ethical problems. The subjects themselves acted as their own controls. Moreover, study was of 6weeks duration thereby a comparison was made only between short-term efficacy and safety of the drugs.

\section{CONCLUSION}

Etoricoxib 30mg once daily and aceclofenac 100mg twice daily were found to be effective in relieving the signs and symptoms of osteoarthritis. Aceclofenac was found to be superior to etoricoxib in some of the parameters of efficacy 
while it was found to be similar in the other efficacy parameters. Both the drug treatments were found to be well tolerated. Adverse effects were observed with aceclofenac as compared to etoricoxib. Thus, absence of adverse drug reactions with etoricoxib $30 \mathrm{mg}$ while exhibiting a good clinical efficacy which was comparable to aceclofenac, a conventional nonsteroidal anti-inflammatory drug makes etoricoxib a useful alternative in patients with osteoarthritis, as NSAIDs are so commonly found to be responsible for adverse effects specifically pertaining to gastrointestinal tract. The findings of the present study need to be proved in further studies involving a larger sample size for a period of more than six weeks. Etoricoxib is a better alternative to conventional NSAIDs for the symptomatic management of osteoarthritis as the safety profile is better. Moreover, etoricoxib is not only safer but found to be clinically efficacious as compared to aceclofenac in the present study.

Funding: No funding sources

Conflict of interest: None declared

Ethical approval: The study was approved by the Institutional Ethics Committee

\section{REFERENCES}

1. Osteoarthritis. Basics Arthritis. CDC. Available at: https://www.cdc.gov/arthritis/basics/osteoarthritis.ht m. Accessed on Dec 27, 2017.

2. WHO. Chronic rheumatic conditions. WHO. Available at: http://www.who.int/chp/topics/rheumatic/en/.

3. Arthritis-India. Available at: http://www.arthritisindia.com/osteoarthritis.html. Accessed on Dec 27, 2017.

4. Diseases and Conditions Osteoarthritis. Available at: https://www.rheumatology.org/I-Am-A/PatientCaregiver/Diseases-Conditions/Osteoarthritis.

5. Osteoarthritis Treatment Information. Available at: https://www.hopkinsarthritis.org/arthritisinfo/osteoarthritis/oa-treatments/. Accessed on Dec 27, 2017.

6. Kasper DL, Harrison TR, editors. Harrison's principles of internal medicine /eds Dennis L. Kasper. Disease pathogenesis and treatment: chapters 99-480. $19^{\text {th }}$ Ed. New York, NY: McGraw Hill Education; 2015(2):467.

7. Goodman LS, Brunton LL, Chabner B, Knollmann BC, editors. Goodman and Gilman's pharmacological basis of therapeutics. $12^{\text {th }}$ Ed. New York: McGrawHill;2011:2084.

8. Hawkey CJ. COX-1 and COX-2 inhibitors. Best Pract Res Clin Gastroenterol. 2001 Oct 1;15(5):801-20.

9. Urban MK. COX-2 specific inhibitors offer improved advantages over traditional NSAIDs. Orthopedics. 2000 Jul;23(7 Suppl):S761-764.

10. Deeks JJ, Smith LA, Bradley MD. Efficacy, tolerability, and upper gastrointestinal safety of celecoxib for treatment of osteoarthritis and rheumatoid arthritis: systematic review of randomised controlled trials. BMJ. 2002 Sep 21;325(7365):619.

11. Bingham CO, Sebba AI, Rubin BR, Ruoff GE, Kremer J, Bird S, et al. Efficacy and safety of etoricoxib $30 \mathrm{mg}$ and celecoxib $200 \mathrm{mg}$ in the treatment of osteoarthritis in two identically designed, randomized, placebocontrolled, non-inferiority studies. Rheumatol Oxf Engl. 2007 Mar;46(3):496-507.

12. Curtis SP, Bockow B, Fisher C, Olaleye J, Compton A, Ko AT, et al. Etoricoxib in the treatment of osteoarthritis over 52-weeks: a double-blind, activecomparator controlled trial (NCT00242489). BMC Musculoskelet Disord. 2005 Dec 1;6:58.

13. Eginster JY, Malmstrom K, Mehta A, Bergman G, Ko AT, Curtis SP, et al. Evaluation of the efficacy and safety of etoricoxib compared with naproxen in two, 138-week randomised studies of patients with osteoarthritis. Ann Rheum Dis. 2007 Jul 1;66(7):94551.

14. Puopolo A, Boice JA, Fidelholtz JL, Littlejohn TW, Miranda P, Berrocal A, et al. A randomized placebocontrolled trial comparing the efficacy of etoricoxib 30 $\mathrm{mg}$ and ibuprofen $2400 \mathrm{mg}$ for the treatment of patients with osteoarthritis. Osteoarthritis Cartilage. 2007 Dec;15(12):1348-56.

15. Stam W, Jansen J, Taylor S. Efficacy of Etoricoxib, celecoxib, lumiracoxib, non-selective NSAIDs, and acetaminophen in osteoarthritis: a mixed treatment comparison. Open Rheumatol J. 2012 Apr 3;6:6-20.

16. Chalini S, Raman U. Comparative efficacy of aceclofenac and etoricoxib in post extraction pain control: randomized control trial. Indian J Dent Res Off Publ Indian Soc Dent Res. 2005 Jun;16(2):47-50.

17. Gottesdiener K, Schnitzer T, Fisher C, Bockow B, Markenson J, Ko A, et al. Results of a randomized, dose-ranging trial of etoricoxib in patients with osteoarthritis. Rheumatol. 2002 Sep 1;41(9):1052-61.

18. Vohra F, Raut A. Comparative efficacy, safety, and tolerability of diclofenac and aceclofenac in musculoskeletal pain management: a systematic review. Indian J Pain. 2016 Jan 1;30(1):3.

19. Batlle-Gualda E, Román Ivorra J, Martín-Mola E, Carbonell Abelló J, Linares Ferrando LF, Tornero Molina $\mathrm{J}$, et al. Aceclofenac vs paracetamol in the management of symptomatic osteoarthritis of the knee: a double-blind 6-week randomized controlled trial. Osteoarthritis Cartilage. 2007 Aug;15(8):900-8.

20. Baraf HSB, Fuentealba C, Greenwald M, Brzezicki J, O'Brien K, Soffer B, et al. Gastrointestinal side effects of etoricoxib in patients with osteoarthritis: results of the etoricoxib versus diclofenac sodium gastrointestinal tolerability and effectiveness (EDGE) trial. J Rheumatol. 2007 Feb;34(2):408-20.

Cite this article as: Waraich HS, Kumar V, Goel A, Singh J. A comparative study to assess the safety and efficacy of etoricoxib versus aceclofenac in osteoarthritis. Int J Basic Clin Pharmacol 2018;7:2010-5. 